Check for updates

1 British Medical Association

2 Dublin, Ireland

JSheather@bma.org.uk

Cite this as: BMJ 2021;375:n2533

http://dx.doi.org/10.1136/bmj.n2533

Published: 18 October 2021

\section{The great resignation-how do we support and retain staff already stretched to their limit?}

\author{
Doctors constantly went above and beyond their ordinary obligations to respond to a threat to \\ national and global wellbeing. But they aren't inexhaustible
}

Julian Sheather, ${ }^{1}$ Dubhfeasa Slattery ${ }^{2}$

After England's “freedom day” and a wave of covid-19 cases this summer, doctors are stoically preparing themselves for a bitter winter. Unlocking during a period of rising case numbers was an unprecedented natural experiment, and the physical harms fell directly on those who became infected. But professional harms have fallen on doctors and other health workers-people already stretched to their limit.

Economists have called the coming months the time of "the great resignation" and our healthcare service is unlikely to emerge unscathed from it. A recent survey of UK doctors found that half of those who responded plan to work fewer hours, with a further $21 \%$ considering leaving the NHS completely. ${ }^{1}$ The number of doctors planning to take early retirement is also on the rise.

Working at the pace required to maintain care standards is becoming impossible and the profession deserves better. Unless the needs of doctors are urgently tackled, medicine is at risk of an eviscerating departure of highly trained clinicians. So, what are the causes of this potential mass exodus, how does it threaten the workforce and patient care, and how can we prevent it?

Burnout has been found to be the factor most strongly related to doctors' plans to withdraw from the clinical workforce. ${ }^{2}$ Of course, it is not a new phenomenon, but surveys have found a growing number of NHS doctors experienced high levels of burnout during the pandemic. ${ }^{3}$ Respondents to staff surveys pointed to workload and the inability to rest as key factors behind their contemplating leaving the NHS. ${ }^{1}$ Eighteen unbroken months of responding to a pandemic have taken a toll. Inadequate personal protective equipment (PPE), moral distress, challenging ethical decisions, colleagues debilitated and dying-all while striving to provide the best patient care-have left many disillusioned and wounded. This cohort of people are often not on the radar of their employers because they keep working to a high standard until they burn out and quietly withdraw from the job, unnoticed by the system.

The silent loss of highly skilled "go-to" colleagues-expert clinicians, mentors, trainers, and key communicators-has a significant impact on patient care, training, career progression, and the workload of colleagues who remain. Loss of this support framework may add to the existing pressure on doctors, leading to increased burnout, more adverse incidents, and greater patient harm.
Critically, the pandemic fell on a workforce that was already under stress: from the burden of working in an environment laden with clinical risk, the pressure of being unable to deliver care to the high standards to which they were trained, and the frustration of chronic under-resourcing and systems failures that result in patient harm.

How should we respond? Good leadership involves anticipating problems. We need to develop and implement a robust organisational framework that supports medical professionalism to improve staff wellbeing and patient centred care.

Healthcare organisations must invest in a range of evidence based interventions to tackle burnout, including individual focused and organisation directed workplace initiatives. The former includes training in stress management, self-care, communication skills, and mindfulness. While the latter includes interventions that improve processes, promote team based care, and reduce clerical burden through use of medical assistants.

Since burnout is associated with medical errors, efforts focused on preventing them will yield twofold benefits for both patient care and staff wellbeing. ${ }^{4}$ Staff training should be expanded to include successful teamwork, communication, and leadership skills in complex situations. Effective pathways must be identified to tackle unprofessional behaviour, along with ongoing real time data analysis of clinical incidents, claims, and reviews to identify overarching lessons and prevent recurrence of adverse incidents.

Different models of peer support programmes also exist that can help clinicians process the emotional fallout of an adverse event. Talking with a peer about a medical error helps doctors cope positively, and the literature shows that doctors prefer one to one peer doctor support (rather than group support), which is free and confidential. Hospitals should consider introducing these programmes. ${ }^{5} 6$

Additionally, we need to introduce a formal mechanism for transitioning senior professionals gradually and partially from full time clinical work to leadership, training, mentoring, and quality and process improvement work. Doctors are expected to perform the same volume of patient-facing clinical work (including overnight on-call) at 55 as they did at 35, yet physically they may be less able to do so. Implementing an organisational system that helps doctors to transition into other areas should help prevent them from retiring early because of clinical workloads; maximise the use of the broad range of 
skills of senior staff; and promote clinician leadership, staff mentoring, and support during adverse events, clinical claims, and referral to regulatory bodies.

Meaningful staff engagement, honest communication, innovation, and clinician leadership from trusted colleagues is invaluable to identify the real problems and inefficiencies in systems, and to codesign practical, solution focused interventions that are acceptable to patient-facing staff, while bridging the gaps that may exist between management and clinicians. The impact of these interventions should be repeatedly and objectively measured to promote learning from excellence and the sharing of successful ideas at a national and international level.

Early in the pandemic, weekly national ovations testified to the dedication, skill, and resourcefulness of health professionals. We saw a nation relying on a deep hinterland of medical professionalism - of people constantly going above and beyond their ordinary obligations to respond to a historic threat to national and global wellbeing. But that hinterland is not inexhaustible. We badly need change.

Competing interests: none declared.

Not commissioned, not peer reviewed

1 Thousands of overworked doctors plan to leave the NHS, BMA finds. www.bma.org.uk/bmamedia-centre/thousands-of-overworked-doctors-plan-to-leave-the-nhs-bma-finds.

2 Sinsky CA, Dyrbye LN, West CP, Satele D, Tutty M, Shanafelt TD. Professional satisfaction and the career plans of US physicians. Mayo Clin Proc 2017;92:1625-35 doi: 10.1016/j.mayocp.2017.08.017 pmid: 29101932

3 Marsh S. One-third of UK trainee doctors feel high level of burnout amid covid. Guardian. 27 July 2021. www.theguardian.com/society/2021/ju//27/one-third-uk-trainee-doctors-feel-high-levelburnout-covid.

4 Shanafelt TD, Balch CM, Bechamps G, etal. Burnout and medical errors among American surgeons. Ann Surg 2010;251:995-1000. doi: 10.1097/SLA.0b013e3181bfdab3 pmid: 19934755

5 Plews-Ogan M, May N, Owens J, Ardelt M, Shapiro J, Bell SK. Wisdom in medicine: what helps physicians after a medical error?Acad Med 2016;91:233-41. doi: 10.1097/ACM.0000000000000886 pmid: 26352764

6 Hu YY, Fix ML, Hevelone ND, etal. Physicians' needs in coping with emotional stressors: the case for peer support. Arch Surg 2012;147:212-7. doi: 10.1001/archsurg.2011.312 pmid: 22106247 